\title{
TRIM28 promotes cervical cancer growth through the mTOR signaling pathway
}

\author{
FAN LI, ZHIJIE WANG and GAOCHUAN LU \\ Department of Gynaecology and Obstetrics, Shanghai Eighth People's Hospital \\ Affiliated to Jiangsu University, Shanghai 200235, P.R. China
}

Received June 15, 2017; Accepted December 12, 2017

DOI: 10.3892/or.2018.6235

\begin{abstract}
Aberrant expression of tripartite motif-containing protein 28 (TRIM28) has been demonstrated in several human cancers; however, its biological function and related mechanism in cervical cancer remain unclear. In this study, we compared TRIM28 expression between cervical cancer and adjacent normal tissues, and detected significant elevation in TRIM28 expression levels in the cervical cancer tissues. Moreover, TRIM28 overexpression promoted the proliferation, colony formation, and cell cycle progression of cervical cancer cell lines, as well as the growth of xenograft tumors in nude mice, whereas knockdown of TRIM28 had the opposite effects. Evaluation of the potential mechanism demonstrated that TRIM28 promoted cervical cancer cell growth by activating the mammalian target of rapamycin (mTOR) signaling pathway. In support of this finding, TRIM28-induced cell proliferation was abolished by treatment with everolimus, a specific mTOR inhibitor. These results suggest that TRIM28 plays a pivotal role in cervical cancer cell proliferation and might serve as a potential therapeutic target.
\end{abstract}

\section{Introduction}

Cervical cancer is one of the most common malignancies in women, with an estimated 530,000 new cases and 275,000 deaths each year worldwide (1). Although mounting studies have demonstrated the importance of human papillomavirus in cervix tumorigenesis $(2,3)$, the detailed mechanism of cervical carcinogenesis remains unclear.

Recent studies have shown that many members of the tripartite motif-containing (TRIM) superfamily are involved in a broad range of biological processes, including immunity (4,5), neurological development (6), cell growth (7), cell differentiation (8), tumorigenesis (9), and responses to

Correspondence to: Dr Fan Li, Department of Gynaecology and Obstetrics, Shanghai Eighth People's Hospital Affiliated to Jiangsu University, 8 Caobao Road, Xuhui, Shanghai 200235, P.R. China E-mail: lifan_shanghai@126.com

Key words: TRIM28, cervical cancer, growth, mTOR microbial infection (10). TRIM proteins are evolutionarily conserved, sharing a common $\mathrm{N}$-terminal really interesting new gene (RING) finger domain, which can mediate the conjugation of proteins with ubiquitin (11). Recently, multiple studies have indicated that some members of the TRIM protein family function as important regulators for carcinogenesis. For example, TRIM13, TRIM19, TRIM24, TRIM25, and TRIM59 were shown to be involved in leukemia, liver, breast, prostate, and gastric cancers, respectively (11-15), demonstrating the crucial roles of the TRIM family in tumorigenesis.

TRIM28 also exhibits certain functions in tumor development (16-18). Yokoe et al (19) found that the expression level of the TRIM28 gene was significantly higher in gastric cancer tissues than in non-cancerous tissues. Patients with high TRIM28 expression showed a higher incidence of peritoneal carcinomatosis and significantly poorer overall survival compared to patients with low TRIM 28 expression. Addison et al (20) showed that TRIM28 is frequently overexpressed in breast tumors at both the mRNA and protein levels; knockdown of TRIM28 in breast cancer cells led to the inhibition of cell proliferation, tumor growth, and metastasis. However, other researchers found that high TRIM28 expression was correlated with increased overall survival in early-stage lung tumors, and TRIM28 overexpression reduced cell proliferation in model lung cancer cell lines (21). These findings suggest that TRIM28 may play a complex role in human cancer.

In the present study, we found that the expression of TRIM28 is greatly increased in cervical cancer tissues and cell lines. In vitro and in vivo experiments further demonstrated that TRIM28 overexpression promotes cervical cancer cell proliferation and xenograft tumor growth, respectively. Further analyses revealed that TRIM28 induced cervical cancer cell growth via the mammalian target of rapamycin (mTOR) signaling pathway.

\section{Materials and methods}

Clinical tissue samples. Twenty paired cervical cancer samples and adjacent normal tissues were obtained from January 2013 to November 2015 at the Shanghai Eighth People's Hospital (Shanghai, China). None of the patients had received chemotherapy, immunotherapy, or radiotherapy before specimen 
collection. All patients were well informed and the process was approved by the Ethics Committee of Eighth People's Hospital affiliated to Jiangsu University, China.

Cell lines and cell culture. The human cervical cancer cell lines SiHa and CaSki were purchased from the American Type Culture Collection (Rockville, MD, USA). The cell lines were cultured in Dulbecco's modified Eagle's medium (Sigma-Aldrich, St. Louis, MO, USA) with $10 \%$ fetal bovine serum (Invitrogen, Carlsbad, CA, USA). The cells were maintained at $37^{\circ} \mathrm{C}$ in a humidified atmosphere containing $5 \% \mathrm{CO}_{2}$.

Lentivirus production. The cDNA of TRIM28 and two short hairpin RNA (shRNA) sequences targeting TRIM28 were separately cloned into lentiviral expression vectors, as described previously (11). The lentiviral vectors were then transfected into $293 \mathrm{~T}$ cells, along with the lentiviral packaging mix. At $48 \mathrm{~h}$ post-transfection, lentiviral supernatants were collected to infect the target cell lines, and a further $48 \mathrm{~h}$ later, infected cells were selected with $8 \mathrm{mg} / \mathrm{ml}$ puromycin for a continuous 2 -week period to generate the stable transfected cell line.

Immunohistochemical staining. Paraffin-embedded tissue blocks were deparaffinized, rehydrated, and subjected to a heat-induced epitope retrieval step in $0.01 \mathrm{M}$ sodium citrate ( $\mathrm{pH}$ 6.0). After blocking the endogenous peroxidase with $3 \%$ hydrogen peroxide, the sections were washed with Tris-buffered saline (TBS) and then incubated with a primary antibody targeting TRIM28 (Proteintech, Hubei, China) for $1 \mathrm{~h}$ at $37^{\circ} \mathrm{C}$. Subsequently, the sections were incubated with a horseradish peroxidase-conjugated secondary antibody for 30 min, developed with 3,3'-diaminobenzidine, and counterstained with hematoxylin.

RNA extraction and quantitative reverse transcription-polymerase chain reaction ( $q R T-P C R)$. Total RNA was extracted from the cervical cancer samples and cell lines, using TRIzol reagent (Invitrogen). cDNA synthesis was performed using PrimeScript RT reagent kit (Takara, Otsu, Japan). qRT-PCR of TRIM28 was conducted using the SYBR Green PCR Master Mix kit (Takara) and ABI 7900HT Fast Real-Time PCR system (Applied Biosystems, Foster City, CA, USA). $\beta$-actin was used as the endogenous normalization control. Data were analyzed by the $2^{-\Delta \Delta \mathrm{Ct}}$ method. All samples were examined in triplicate.

Cell viability analysis. To test cell viability, $2 \times 10^{3}$ cells were cultured in 96-well plates for 24, 48, 72 and $96 \mathrm{~h}$, and 3-(4,5-dimethyl-2-thiazolyl)-2,5-diphenyl-2H-tetrazolium bromide (MTT) assays were performed according to the manufacturer's protocol (Invitrogen). In brief, the cells were stained with $100 \mu \mathrm{l} \mathrm{MTT}(0.5 \mathrm{mg} / \mathrm{ml})$ for $4 \mathrm{~h}$ at $37^{\circ} \mathrm{C}$, followed by removal of the culture medium and addition of $150 \mu \mathrm{l}$ dimethyl sulfoxide. The absorbance was measured at $490 \mathrm{~nm}$.

Colony formation assay. The cells were plated in a 6-well plate containing 500 cells per well in triplicate. After 14 days, the cells were stained with $0.005 \%$ crystal violet (dissolved in methanol) for $1.5 \mathrm{~h}$, and then counted and photographed.

Cell cycle analysis. Cells $\left(1 \times 10^{6}\right)$ were harvested and washed with cold phosphate-buffered saline (PBS), followed by fixation with $70 \%$ ethanol for $24 \mathrm{~h}$ at $4^{\circ} \mathrm{C}$. After washing with PBS, the cells were incubated with propidium iodide (Sigma-Aldrich) and DNase-free RNase for $30 \mathrm{~min}$ at room temperature. The cell cycle was measured using a FACSCalibur flow cytometer (BD Biosciences, San Jose, CA, USA) with CellQuest software.

Bromodeoxyuridine (BrdU) incorporation assay. The BrdU assay was performed as previously described (22). In brief, cells grown on coverslips (Fisher, Pittsburgh, PA, USA) were incubated with BrdU for $1 \mathrm{~h}$ and stained with anti-BrdU antibody (Upstate, Temecula, CA, USA) according to the manufacturer's instructions. Gray-level images were acquired under a laser-scanning microscope (Axioskop 2 Plus, Carl Zeiss Co., Ltd., Jena, Germany).

Tumor xenograft experiment. Female BALB/c-nude mice (4-6 weeks old) were purchased from Shanghai Laboratory Animal Center (Shanghai, China). All experimental procedures were approved by the Institutional Animal Care and Use Committee of Eighth People's Hospital affiliated to Jiangsu University, China. SiHa cells $\left(2 \times 10^{6}\right.$ cells/mouse) and CaSki cells $\left(5 \times 10^{6}\right.$ cells/mouse) were injected subcutaneously into the nude mice ( $\mathrm{n}=5$ per group). Tumor volume (V) was monitored every 4 days and calculated using the formula: $\mathrm{V}=0.5 \mathrm{x}$ length $\mathrm{x}$ width $^{2}$. At the end of the experiment, the mice were sacrificed and tumor weights were assessed. The proliferation index was determined by $\mathrm{Ki} 67$ immunostaining and calculating the ratio of Ki67-positive cells among the total number of cells in five randomly selected fields.

Western blot analysis. The cells were lysed with RIPA buffer supplemented with protease and phosphatase inhibitors (Roche Diagnostics, Branchburg, NJ, USA). The protein concentration was measured by the BCA Protein assay (Bio-Rad Laboratories, Hercules, CA, USA). Proteins were separated by $10 \%$ sodium dodecyl sulfate-polyacrylamide gel electrophoresis and transferred onto polyvinylidene fluoride membranes (Millipore, Billerica, MA, USA). The membranes were blocked with $5 \%$ bovine serum albumin in TBS for $1 \mathrm{~h}$ at room temperature, and then incubated with the primary antibodies against TRIM28 (15202-1-AP) and GAPDH (10494-1-AP) (both from Proteintech) overnight at $4^{\circ} \mathrm{C}$. The membranes were then washed in TBS containing $1 \%$ Tween-20, incubated with the horseradish peroxidase-conjugated secondary antibody for $1 \mathrm{~h}$ at room temperature, and developed by ECL reagent (Pierce, Rockford, IL, USA).

Statistical analysis. All experiments were performed at least in triplicate and the data are presented as the means \pm standard deviations. All statistical analyses were carried out using the SPSS 16.0 statistical software package (SPSS, Inc., Chicago, IL, USA). The two-sided Student's t-test was performed to assess differences between groups. $\mathrm{P}<0.05$ was considered statistically significant. 

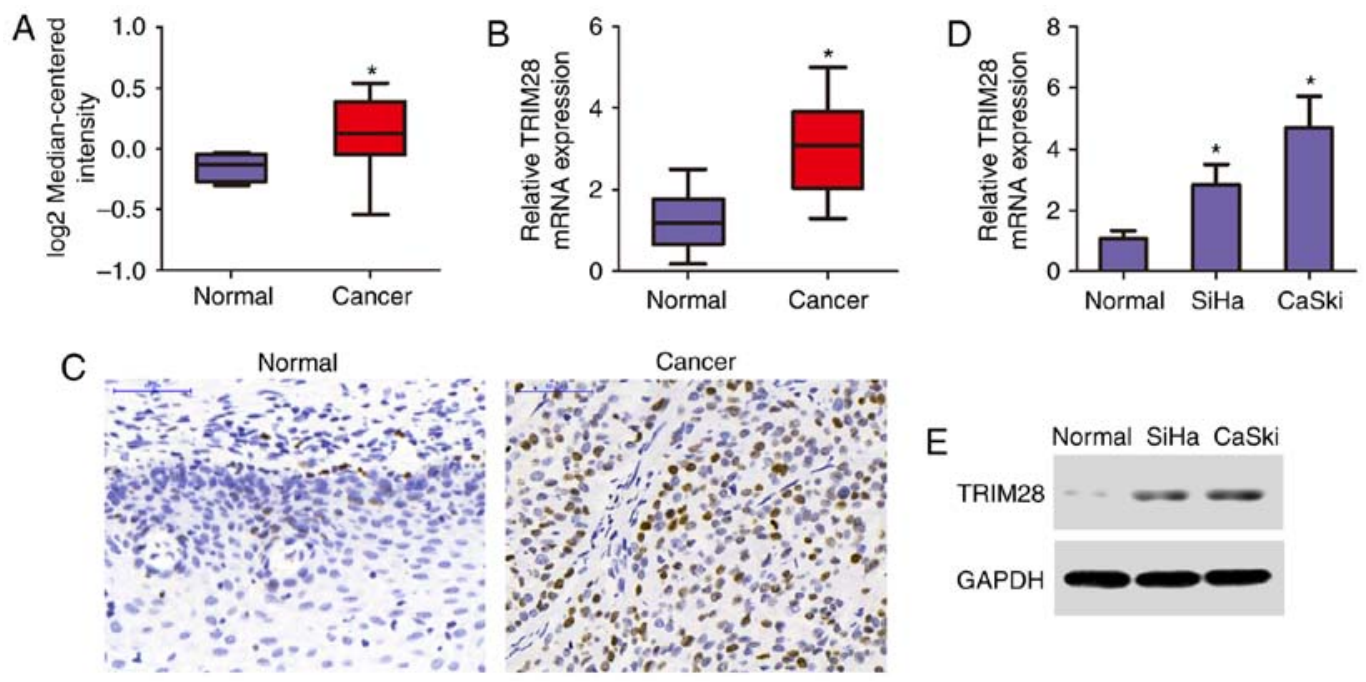

Figure 1. TRIM28 expression is elevated in cervical cancer tissues and cell lines. (A) Analysis from the Oncomine database showing that TRIM28 mRNA expression is significantly elevated in cervical cancer tissues $(\mathrm{n}=40)$ compared with that in normal tissues $(\mathrm{n}=5)$. The data were obtained from a previous study (35). (B) TRIM28 mRNA levels in 20 paired cervical cancer samples and adjacent normal tissues examined by qRT-PCR. (C) Immunohistochemical staining of normal and cervical cancer tissues with anti-TRIM28 antibody. The expression levels of TRIM28 in human cervical cancer cells and normal human cervical tissues were measured by qRT-PCR (D) and western blotting (E). Data are presented as means \pm SD. ${ }^{*} \mathrm{P}<0.05$.

\section{Results}

TRIM28 expression is greatly increased in cervical cancer tissues and cell lines. To determine the expression of TRIM28 in cervical cancer, we first analyzed related data in the Oncomine database, which revealed marked elevation in TRIM28 expression levels in cervical cancer tissues compared with that in normal tissues (Fig. 1A). We next tested the relative expression levels of TRIM28 in 20 cervical cancer samples and paired adjacent normal tissues by qRT-PCR. The results indicated that the TRIM 28 expression was significantly upregulated in cervical cancer tissues compared with that in the adjacent normal tissues (Fig. 1B). Moreover, the protein level of TRIM28 was increased in the cervical cancer samples, as shown by immunohistochemistry (Fig. 1C). Consistent with these observations, both the mRNA and protein levels of TRIM28 were differentially upregulated in the cervical cancer cell lines SiHa and CaSki compared with that in the human normal cervical tissues (Fig. 1D and E), suggesting that TRIM28 is upregulated in cervical cancer tissues and cell lines.

TRIM28 promotes proliferation of cervical cancer cells by accelerating the cell cycle. To examine the biological role of TRIM28 in cervical cancer, the cervical cancer cell lines $\mathrm{SiHa}$ and CaSki were established to stably express TRIM 28 or TRIM 28 shRNA, using a lentivirus-mediated overexpression or knockdown system, respectively (Fig. 2A). MTT and colony formation assays showed that ectopic expression of TRIM28 dramatically increased cell growth, whereas depletion of TRIM28 expression reduced the growth rate of cervical cancer cell lines compared with that of control cells (Fig. 2B and C). Flow cytometry analysis revealed that the TRIM28-overexpressing cells showed a significant increase in the percentage of cells in the S peak and a decrease in the percentage of cells in the G0-G1 peak compared with control cells, whereas knockdown of TRIM28 had the opposite effect on CaSki cells (Fig. 2D), indicating that the effect of TRIM28 on cell proliferation might be due to regulation of the G1-S phase transition. In addition, BrdU incorporation was markedly increased by the ectopic expression of TRIM28 in $\mathrm{SiHa}$ cells, and was suppressed by TRIM28 silencing in CaSki cells (Fig. 2E). These results further supported the notion that upregulation of TRIM28 regulates the G1-S phase transition of cervical cancer cells.

TRIM28 promotes tumor formation of cervical cancer cells in vivo. We further evaluated the effect of TRIM 28 on the tumorigenic activity of cervical cancer cells. As shown in Fig. 3A and B, the TRIM28-overexpressing tumors grew at a much higher rate, as determined by size and weight, than the control tumors, whereas the tumors formed by TRIM28-silenced cells were smaller and had lower tumor weights than those formed from shRNA-vector control cells. Immunohistochemistry analysis revealed that TRIM28-overexpressing tumors displayed a higher Ki67 proliferation index, whereas the TRIM28-silenced tumors showed reduced numbers of Ki67positive cells (Fig. 3C). Protein expression levels of TRIM28 in xenografts were further examined by western blotting. TRIM28 was robustly upregulated in tumors formed by TRIM28-overexpressing SiHa cells, but was downregulated in tumors formed by TRIM28-silencing CaSki cells (Fig. 3D). Taken together, these results suggest that TRIM28 promotes the tumorigenicity of cervical cancer cells in vivo.

TRIM28 stimulates cervical cancer cell proliferation via the mTOR signaling pathway. As mTOR is a central regulator of the cell cycle, cell growth, and proliferation $(23,24)$, we investigated whether the TRIM28-induced cervical cancer cell proliferation was mediated through the mTOR signaling pathway. Western blot analyses showed that downregulation of TRIM28 significantly inhibited the phosphorylation of mTOR and its downstream molecule S6K1, while upregulation of TRIM28 increased the activity of mTOR/S6K1 

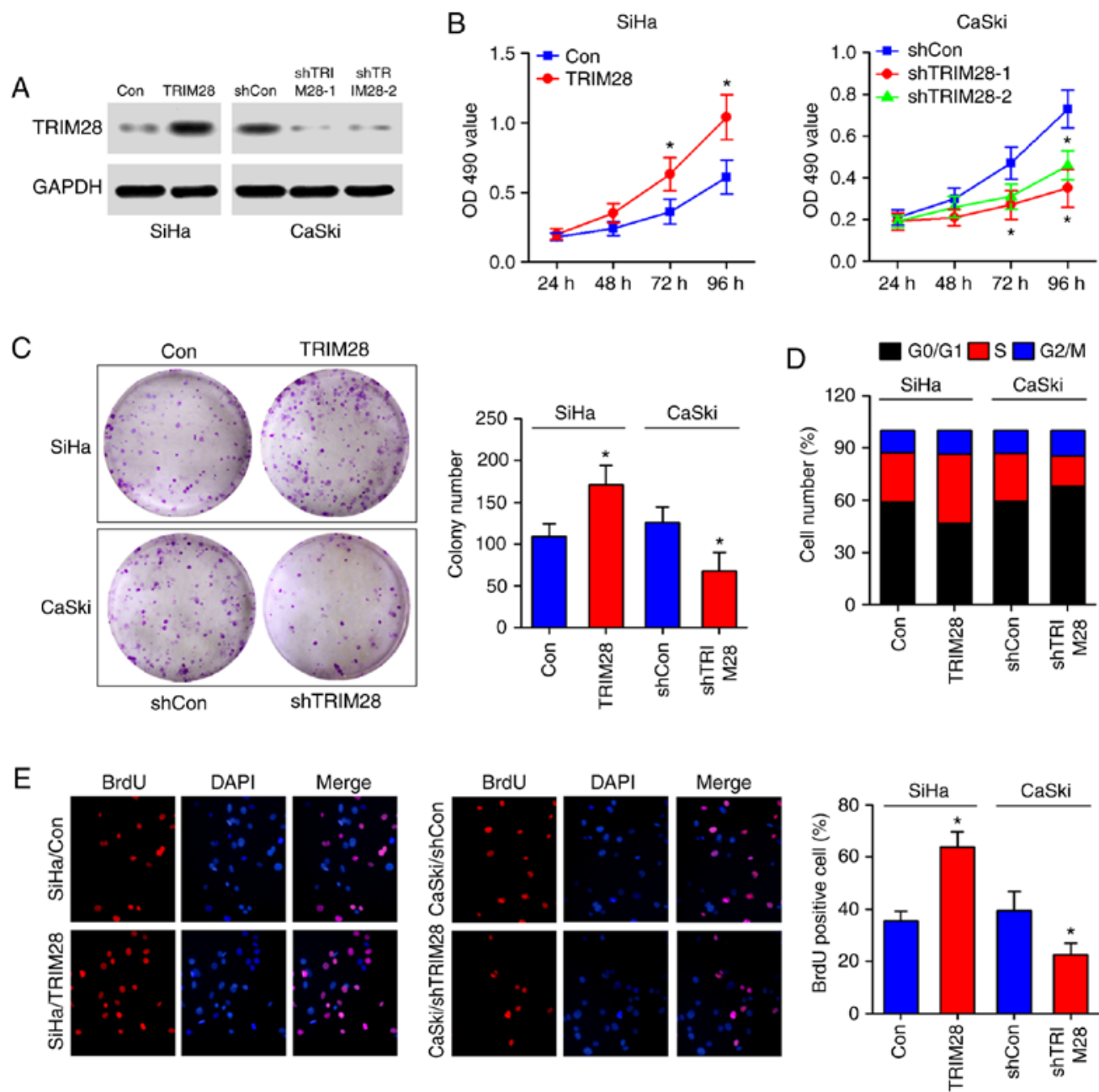

Figure 2. TRIM28 promotes cervical cancer cell proliferation by accelerating the cell cycle. (A) Western blot analysis of TRIM28 protein expression in TRIM28-infected cells and TRIM28 shRNA-infected cells. GAPDH was used as the loading control. (B) Effects of TRIM28 upregulation or downregulation on the proliferation of SiHa or CaSki cells analyzed by the MTT assay. (C) Representative micrographs (left) and quantification (right) of colonies formed by the indicated cervical cancer cell lines. (D) Cell cycle progression of the indicated cervical cancer cell lines analyzed by flow cytometry. (E) Representative images (left) and quantification (right) of BrdU-positive cells in the indicated cervical cancer cell lines. Data are presented as means $\pm \mathrm{SD}$. ${ }^{*} \mathrm{P}<0.05$.
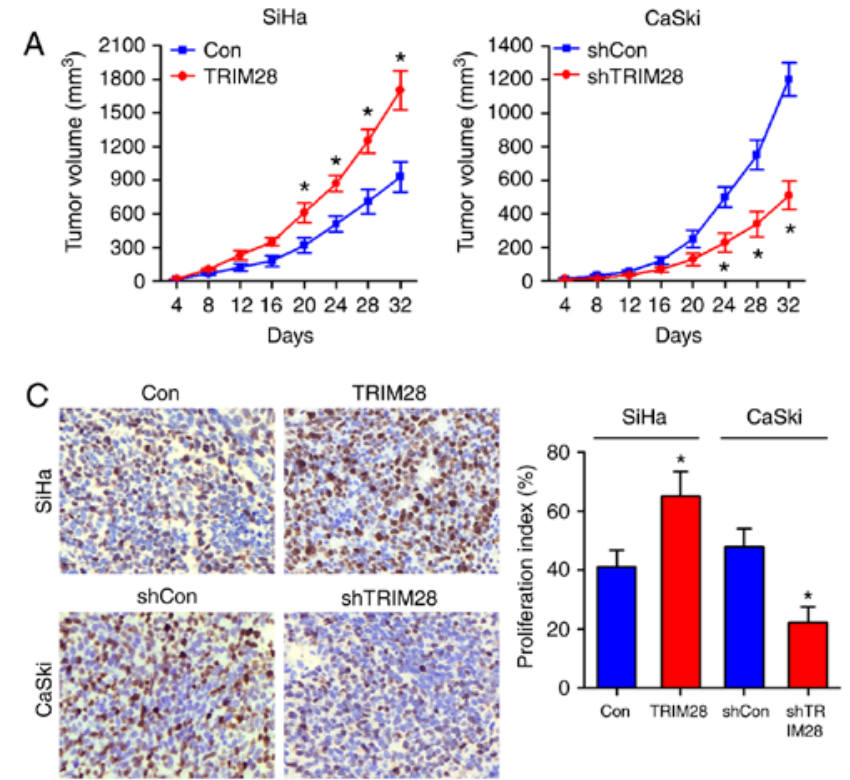
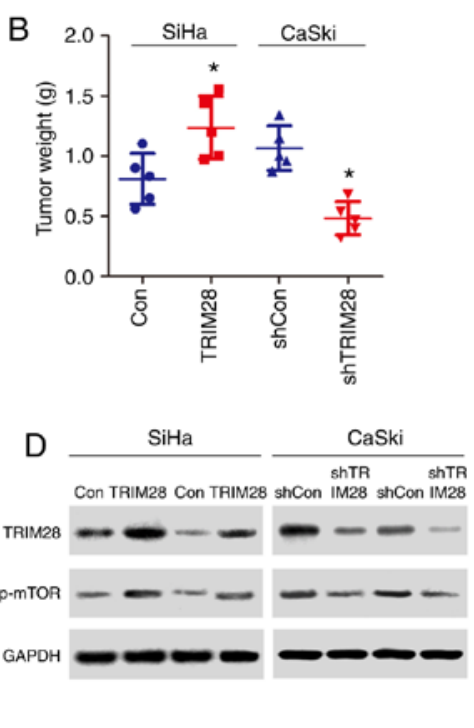

Figure 3. TRIM28 enhances cervical cancer cell tumorigenicity. (A) Tumor volumes were measured on the indicated days. (B) At the end of the experiment, the weights were measured. (C) The proliferation index (right) was determined based on the percentage of Ki67-positive cells (left). (D) Western blot analysis of TRIM28 and p-mTOR protein expression in indicated tumor xenografts. Data are presented as means $\pm \mathrm{SD}$. ${ }^{*} \mathrm{P}<0.05$. 

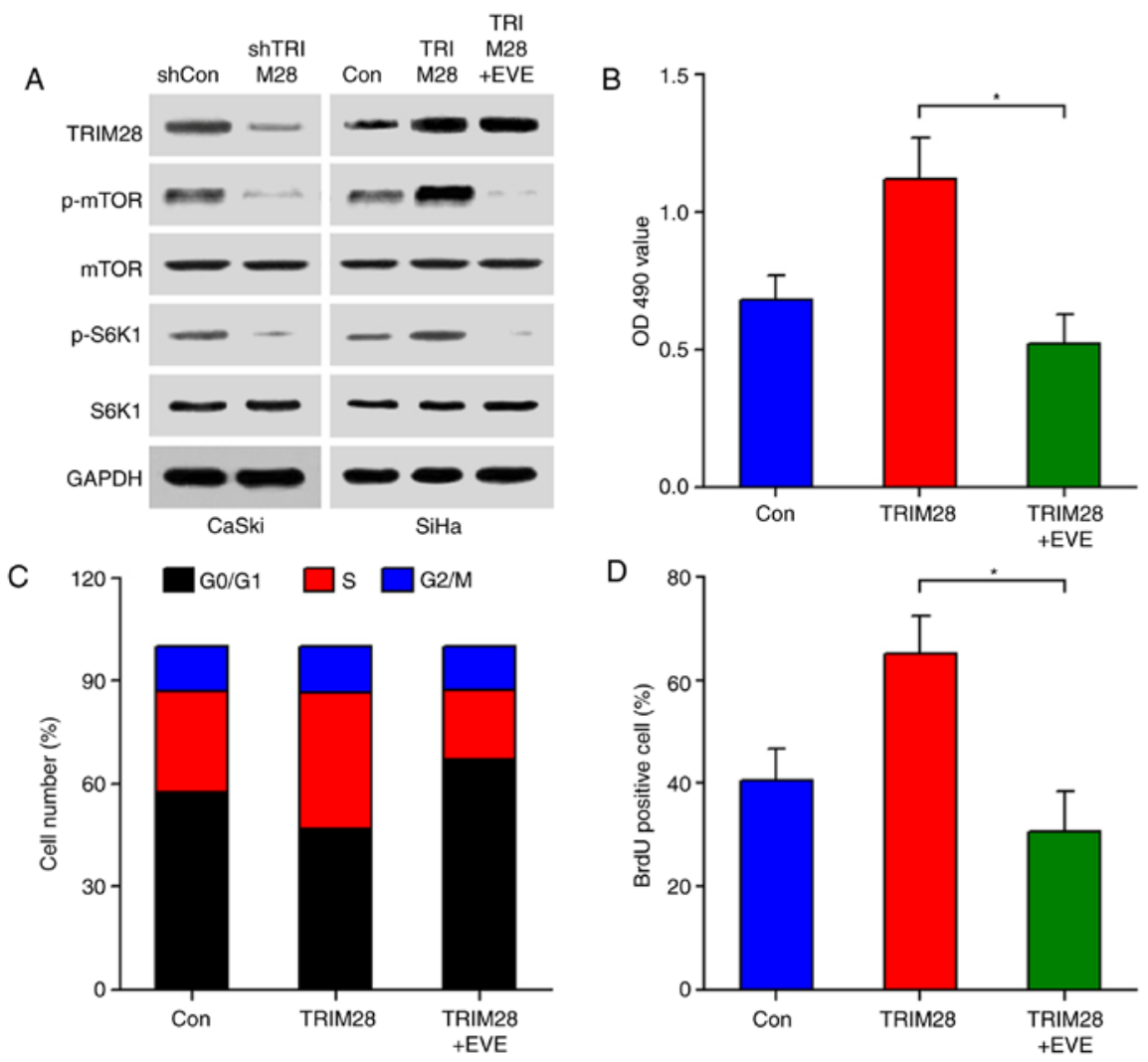

Figure 4. TRIM28 promotes cervical cancer cell proliferation via the mTOR signaling pathway. (A) Western blot analysis of TRIM28, p-mTOR(S2448), mTOR, p-S6K1(T389), and S6K1 in TRIM28 shRNA-infected CaSki cells and TRIM28-infected SiHa cells in the presence or absence of $20 \mathrm{nM}$ everolimus (EVE) for $48 \mathrm{~h}$. GAPDH served as the loading control. (B-D) Cell proliferation, cell cycle, and BrdU incorporation were analyzed in TRIM28-infected $\mathrm{SiHa}$ cells in the presence or absence of EVE for $48 \mathrm{~h}$. Data are presented as means $\pm \mathrm{SD}$. ${ }^{*} \mathrm{P}<0.05$.

signaling. However, modulation of TRIM28 did not alter the total protein levels of mTOR and S6K1 (Fig. 4A). TRIM28modulated activation of mTOR was also confirmed in tumor xenografts (Fig. 3D). We next examined the effect of TRIM28 upregulation on the mTOR signaling pathway in the presence of everolimus, a specific mTOR inhibitor. As shown in Fig. 4A, everolimus significantly abrogated the TRIM28-induced phosphorylation of mTOR and S6K1. In line with this, TRIM28-induced cell proliferation was also abolished by everolimus, as determined by MTT, cell cycle, and BrdU incorporation assays (Fig. 4B-D). Collectively, our results indicate that TRIM28 promotes cervical cancer cell proliferation through activating the mTOR signaling pathway.

\section{Discussion}

Many members of the TRIM family have been shown to play pivotal roles in tumorigenesis. TRIM28 is one of 60 members of the TRIM family. To date, the biological functions and molecular mechanisms of TRIM28 in cervical cancer remain unclear. In this study, we found that the expression of TRIM 28 is significantly upregulated in cervical cancer tissues and cell lines compared with that in their normal counterparts. Moreover, TRIM28 promoted cervical cancer cell proliferation by activating the mTOR signaling pathway. Thus, our findings may provide a novel target for the therapeutic intervention of cervical cancer.
TRIM28, also known as Krüppel-associated box (KRAB)associated protein 1 (KAP1) or transcription intermediary factor 1 (TIF1 $\beta$ ), is a universal co-repressor for KRAB family zinc finger proteins (KRAB-ZNF) (25). TRIM28 has multifaceted roles in many organismal processes. Mouse embryos deficient in TRIM28 develop normally to the blastocyst stage and can implant but fail to gastrulate, indicating that TRIM 28 is essential for early post-implantation mouse development (26). TRIM28 is also critical for maintenance of the pluripotent state of embryonic stem cells (27). Recently, TRIM28 was reported to regulate autophagy, a stress-induced process that degrades subcellular constituents (18). Some researchers have demonstrated that TRIM28 is significantly upregulated in various types of tumors, including breast, liver, brain, and ovarian cancers $(28,29)$, and contributes to tumor progression. However, the underlying cellular functions of TRIM28 and the related mechanisms involved in its carcinogenicity remain largely unexplored. Lin et al (30) identified TRIM28 as a potential marker of cervical cancer metastasis; increase in TRIM28 expression enhanced the migration and invasion of cervical cancer cells both in vitro and in vivo. In the present study, we also detected the elevated expression of TRIM28 in cervical cancer tissues relative to that in matched normal tissues. Importantly, TRIM28 overexpression promoted the proliferation, clone formation, and cell cycle progression of cervical cancer cell lines, as well as the growth of xenograft tumors in nude mice. Conversely, knockdown of TRIM28 reduced cell proliferation and tumor growth. The present 
results clearly indicate that TRIM28 is involved in cervical cancer cell proliferation. However, further investigations are needed to explore the underlying mechanisms involved in these TRIM28-mediated effects.

The conserved serine/threonine kinase mTOR is a key downstream effector of several signaling pathways involved in cancer progression, including the phosphoinositide 3-kinase, mitogen-activated protein kinase, and AMP-activated protein kinase pathways $(24,31)$. Abnormal activation of mTOR signaling occurs in different types of tumors, including cervical cancer (32). For example, through the post-transcriptional regulation of mTOR expression, La-related protein 1 (LARP1) contributes to cervical cancer progression and adverse prognosis (33). Inhibition of mTOR signaling with rapamycin decreased the progranulin-stimulated protein synthesis, transformation, and proliferation of cervical cells in vitro, as well as tumor formation and growth in vivo, suggesting that the activated mTOR pathway is critical for cervical cancer cell growth, proliferation, and survival (34). Therefore, we investigated whether mTOR signaling is involved in the TRIM28-induced carcinogenesis of cervical cancer. We found that upregulation of TRIM28 significantly increased the phosphorylation of mTOR and its downstream molecule S6K1, while downregulation of TRIM28 inhibited the activity of mTOR/S6K1 signaling. TRIM28-modulated activation of mTOR was also confirmed in tumor xenografts. Furthermore, we evaluated the effects of everolimus on TRIM28-overexpressing cervical cancer cells. The results showed that treatment with $20 \mathrm{nM}$ everolimus significantly abrogated the TRIM28-induced phosphorylation of mTOR/S6K1 and cervical cancer cell proliferation, suggesting that mTOR may be the main downstream effector of TRIM28 in promoting cervical cancer growth.

In conclusion, this study revealed that TRIM28 plays an essential role in the progression of cervical cancer, and thus might represent a new therapeutic target for cervical cancer.

\section{References}

1. Jemal A, Bray F, Center MM, Ferlay J, Ward E and Forman D: Global cancer statistics. CA Cancer J Clin 61: 69-90, 2011.

2. Walboomers JM, Jacobs MV, Manos MM, Bosch FX, Kummer JA, Shah KV, Snijders PJ, Peto J, Meijer CJ and Muñoz N: Human papillomavirus is a necessary cause of invasive cervical cancer worldwide. J Pathol 189: 12-19, 1999.

3. Garland SM, Hernandez-Avila M, Wheeler CM, Perez G, Harper DM, Leodolter S, Tang GW, Ferris DG, Steben M, Bryan J, et al; Females United to Unilaterally Reduce Endo/ Ectocervical Disease (FUTURE) I Investigators: Quadrivalent vaccine against human papillomavirus to prevent anogenital diseases. N Engl J Med 356: 1928-1943, 2007.

4. Ozato K, Shin DM, Chang TH and Morse HC III: TRIM family proteins and their emerging roles in innate immunity. Nat Rev Immunol 8: 849-860, 2008.

5. Gallouet AS, Ferri F, Petit V, Parcelier A, Lewandowski D, Gault N, Barroca V, Le Gras S, Soler E, Grosveld F, et al: Macrophage production and activation are dependent on TRIM33. Oncotarget 8: 5111-5122, 2017.

6. Song S, Ge Q, Wang J, Chen H, Tang S, Bi J, Li X, Xie Q and Huang X: TRIM-9 functions in the UNC-6/UNC-40 pathway to regulate ventral guidance. J Genet Genomics 38: 1-11, 2011.

7. Raheja R, Liu Y, Hukkelhoven E, Yeh N and Koff A: The ability of TRIM3 to induce growth arrest depends on RING-dependent E3 ligase activity. Biochem J 458: 537-545, 2014

8. Schwamborn JC, Berezikov E and Knoblich JA: The TRIM-NHL protein TRIM32 activates microRNAs and prevents self-renewal in mouse neural progenitors. Cell 136: 913-925, 2009.
9. Yin J, Kim TH, Park N, Shin D, Choi HI, Cho S, Park JB and Kim JH: TRIM71 suppresses tumorigenesis via modulation of Lin28B-let-7-HMGA2 signaling. Oncotarget 7: 79854-79868, 2016.

10. Huang Y, Yu Y, Yang Y, Yang M, Zhou L, Huang X and Qin Q: Fish TRIM8 exerts antiviral roles through regulation of the proinflammatory factors and interferon signaling. Fish Shellfish Immunol 54: 435-444, 2016.

11. Zhou Z, Ji Z, Wang Y,Li J, Cao H, Zhu HH and Gao WQ: TRIM59 is up-regulated in gastric tumors, promoting ubiquitination and degradation of p53. Gastroenterology 147: 1043-1054, 2014.

12. Tyybakinoja A, Vilpo J and Knuutila S: High-resolution oligonucleotide array-CGH pinpoints genes involved in cryptic losses in chronic lymphocytic leukemia. Cytogenet Genome Res 118: 8-12, 2007.

13. Chung YL and Wu ML: Promyelocytic leukaemia protein links DNA damage response and repair to hepatitis B virus-related hepatocarcinogenesis. J Pathol 230: 377-387, 2013.

14. Tsai WW, Wang Z, Yiu TT, Akdemir KC, Xia W, Winter S, Tsai CY, Shi X, Schwarzer D, Plunkett W, et al: TRIM24 links a non-canonical histone signature to breast cancer. Nature 468: 927-932, 2010.

15. Wang S, Kollipara RK, Humphries CG, Ma SH, Hutchinson R, Li R, Siddiqui J, Tomlins SA, Raj GV and Kittler R: The ubiquitin ligase TRIM25 targets ERG for degradation in prostate cancer. Oncotarget 7: 64921-64931, 2016.

16. Herquel B, Ouararhni K, Khetchoumian K, Ignat M, Teletin M, Mark M, Béchade G, Van Dorsselaer A, Sanglier-Cianférani S, Hamiche A, et al: Transcription cofactors TRIM24, TRIM28, and TRIM33 associate to form regulatory complexes that suppress murine hepatocellular carcinoma. Proc Natl Acad Sci USA 108: 8212-8217, 2011.

17. Kim WJ, Wittner BS, Amzallag A, Brannigan BW, Ting DT, Ramaswamy S, Maheswaran S and Haber DA: The WTX tumor suppressor interacts with the transcriptional corepressor TRIM28. J Biol Chem 290: 14381-14390, 2015.

18. Pineda CT and Potts PR: Oncogenic MAGEA-TRIM28 ubiquitin ligase downregulates autophagy by ubiquitinating and degrading AMPK in cancer. Autophagy 11: 844-846, 2015.

19. Yokoe T, Toiyama Y, Okugawa Y, Tanaka K, Ohi M, Inoue Y, Mohri Y, Miki C and Kusunoki M: KAP1 is associated with peritoneal carcinomatosis in gastric cancer. Ann Surg Oncol 17: 821-828, 2010

20. Addison JB, Koontz C, Fugett JH, Creighton CJ, Chen D, Farrugia MK, Padon RR, Voronkova MA, McLaughlin SL, Livengood RH, et al: KAP1 promotes proliferation and metastatic progression of breast cancer cells. Cancer Res 75: 344-355, 2015.

21. Chen L, Chen DT, Kurtyka C, Rawal B, Fulp WJ, Haura EB and Cress WD: Tripartite motif containing 28 (Trim28) can regulate cell proliferation by bridging HDAC1/E2F interactions. J Biol Chem 287: 40106-40118, 2012.

22. Lin C, Wu Z, Lin X, Yu C, Shi T, Zeng Y, Wang X, Li J and Song L: Knockdown of FLOT1 impairs cell proliferation and tumorigenicity in breast cancer through upregulation of FOXO3a. Clin Cancer Res 17: 3089-3099, 2011.

23. Fingar DC, Richardson CJ, Tee AR, Cheatham L, Tsou C and Blenis J: mTOR controls cell cycle progression through its cell growth effectors S6K1 and 4E-BP1/eukaryotic translation initiation factor 4E. Mol Cell Biol 24: 200-216, 2004.

24. Pópulo H, Lopes JM and Soares P: The mTOR signalling pathway in human cancer. Int J Mol Sci 13: 1886-1918, 2012.

25. Czerwińska P, Shah PK, Tomczak K, Klimczak M, Mazurek S, Sozańska B, Biecek P, Korski K, Filas V, Mackiewicz A, et al: TRIM28 multi-domain protein regulates cancer stem cell population in breast tumor development. Oncotarget 8: 863-882, 2017.

26. Cammas F, Mark M, Dollé P, Dierich A, Chambon P and Losson R: Mice lacking the transcriptional corepressor TIF1beta are defective in early postimplantation development. Development 127: 2955-2963, 2000.

27. Seki Y, Kurisaki A, Watanabe-Susaki K, Nakajima Y, Nakanishi M, Arai Y, Shiota K, Sugino H and Asashima M: TIFlbeta regulates the pluripotency of embryonic stem cells in a phosphorylation-dependent manner. Proc Natl Acad Sci USA 107: 10926-10931, 2010

28. Wei C, Cheng J, Zhou B, Zhu L, Khan MA, He T, Zhou S, He J, Lu X, Chen H, et al: Tripartite motif containing 28 (TRIM28) promotes breast cancer metastasis by stabilizing TWIST1 protein. Sci Rep 6: 29822, 2016. 
29. Wang Y, Jiang J, Li Q, Ma H, Xu Z and Gao Y: KAP1 is overexpressed in hepatocellular carcinoma and its clinical significance. Int J Clin Oncol 21: 927-933, 2016.

30. Lin LF, Li CF, Wang WJ, Yang WM, Wang DD, Chang WC, Lee WH and Wang JM: Loss of ZBRK1 contributes to the increase of KAP1 and promotes KAP1-mediated metastasis and invasion in cervical cancer. PLoS One 8: e73033, 2013.

31. Pineda CT, Ramanathan S, Fon Tacer K, Weon JL, Potts MB, $\mathrm{Ou}$ YH, White MA and Potts PR: Degradation of AMPK by a cancer-specific ubiquitin ligase. Cell 160: 715-728, 2015.

32. Molinolo AA, Marsh C, El Dinali M, Gangane N, Jennison K, Hewitt S, Patel V, Seiwert TY and Gutkind JS: mTOR as a molecular target in HPV-associated oral and cervical squamous carcinomas. Clin Cancer Res 18: 2558-2568, 2012.
33. Mura M, Hopkins TG, Michael T, Abd-Latip N, Weir J, Aboagye E, Mauri F, Jameson C, Sturge J, Gabra H, et al: LARP1 post-transcriptionally regulates mTOR and contributes to cancer progression. Oncogene 34: 5025-5036, 2015.

34. Feng T, Zheng L, Liu F, Xu X, Mao S, Wang X, Liu J, Lu Y, Zhao W, Yu X, et al: Growth factor progranulin promotes tumorigenesis of cervical cancer via PI3K/Akt/mTOR signaling pathway. Oncotarget 7: 58381-58395, 2016.

35. Biewenga P, Buist MR, Moerland PD, Ver Loren van Themaat E, van Kampen AH, ten Kate FJ and Baas F: Gene expression in early stage cervical cancer. Gynecol Oncol 108: 520-526, 2008. 